\title{
PERFIL DE LOS HOGARES ECUATORIANOS Y SUS PRÁCTICAS AMBIENTALES. UN ANÁLISIS MULTIDIMENSIONAL
}

\author{
Leonardo Antonino Ávila Proaño; Gladys Janett Buitrón Sánchez; Edwin Javier Suquillo Guijarro \\ ${ }^{1}$ Pontificia Universidad Católica del Ecuador, Facultad de Ciencias Administrativas y Contables, Quito-Ecuador \\ ${ }^{2}$ Pontificia Universidad Católica del Ecuador, Facultad de Ciencias Exactas, Escuela de Física y Matemáticas, Quito-Ecuador \\ *Autor para correspondencia: e-mail: gbuitron860@puce.edu.ec \\ Recibido: 2016/25/10Ａprobado: 2018/05/06
}

DOI: https://doi.org/10.26621/XIV18.2018.06.A07.PUCESI.2550.6684

\begin{abstract}
RESUMEN
El propósito de la investigación es determinar el perfil de los hogares ecuatorianos; respecto a los hábitos y sus prácticas ambientales. Se utilizó la base de datos de la encuesta nacional Prácticas Ambientales en los Hogares, realizada por el INEC el 2015. Se efectuó el análisis estadístico descriptivo, análisis factorial de correspondencias y análisis jerárquico para definir las clases de hogares. Las variables que describen los ejes factoriales son: área de ubicación, clasificación de los residuos, forma de eliminar los residuos, hábitos e instalaciones para ahorro del agua. Los principales resultados son: el 59.2\% de hogares no clasifica los residuos. El 33.1\% de hogares depositaron los desechos electrónicos/ eléctricos con el resto de basura, el $47.5 \%$ eliminó el aceite y/o grasas de cocina con el resto de basura y el $69.6 \%$ eliminó los focos ahorradores con el resto de basura. El 76.3\% de hogares cierran las llaves mientras jabonan los platos, se bañan y se cepillan los dientes. Solo el $7.7 \%$ de hogares disponen de economizadores de chorro, el $4 \%$ disponen de inodoro doble descarga y el $3 \%$ colocan una botella de agua u otro objeto dentro del tanque. El primer grupo jerárquico (36.93\% de hogares) es el más representativo, cuyo perfil es: hogares del área urbana, no clasifican los residuos porque no hay contenedores específicos o centros de acopio para reciclables, depositan con el resto de basura aceites y grasas de cocina y focos ahorradores.
\end{abstract}

Palabras clave: buenas prácticas ambientales, estadística descriptiva, estadística multidimensional.

\begin{abstract}
The purpose of the research is to determine the profile of Ecuadorian households regarding environmental habits and practices. In order to do so, the database of the national survey on Household Environmental Practices, carried out by the INEC in 2015, was used. With the aim of defining the types of existing households, a descriptive statistical analysis, a correspondence factor analysis and a hierarchical analysis were carried out. The variables that describe the factorial axes are: location area, classification of waste, way of eliminating waste, habits and facilities for saving water. The main results showed that $59.2 \%$ of households do not classify waste; $33.1 \%$ of households throw the electronic / electrical waste away together with the rest of garbage; $47.5 \%$ eliminate the oil and / or cooking fats with the rest of garbage and $69.6 \%$ eliminate the saving lights with the rest of garbage; $76.3 \%$ of households turn off the tap while they soap up the dishes, have a bath and brush their teeth. Only $7.7 \%$ of homes have waterside economizers, $4 \%$ have double flush toilets and $3 \%$ place a water bottle or other object inside the toilet
\end{abstract}


tank. The first hierarchical group (36.93\% of households) is the most representative one, whose houses are in the urban area, therefore, they do not sort the waste since they do not have specific containers or collection centers for recyclables, they deposit oils, fats and saving lights with the rest of the garbage.

Keywords: good environmental practices, descriptive statistics, multidimensional statistics.

\section{INTRODUCCIÓN}

La presente investigación propone una visión de los hogares ecuatorianos y sus prácticas ambientales en relación al área geográfica, clasificación y forma de eliminación de los residuos, prácticas en el manejo del agua y energía eléctrica, consumo responsable y participación en actividades a favor del ambiente. Las buenas prácticas ambientales comprenden un proceso de concientización sobre la limitación de la disponibilidad de los recursos naturales, desde el nivel individual hasta el comunitario (CEPAL, 2015; ONU, 2013; PNUMA, 2011).

La Comisión Económica para América Latina y el Caribe (CEPAL), desde la década 1970, ha apoyado el tema ambiental (INEC, 2015), muestra de ello es la conferencia de las Naciones Unidas sobre el Desarrollo Sostenible Río+20 y la agenda de los Objetivos de Desarrollo del Milenio (CEPAL, 2015). De igual forma, Naciones Unidas organizó la Tercera Conferencia sobre Vivienda y Desarrollo Sostenible-Hábitat III, que tuvo lugar en Quito en octubre de 2016, la que congregó a gobernantes, alcaldes, actores urbanos del sector público y privado y sociedad civil, con el propósito de convenir estrategias, soluciones y compromisos a nivel político para el desarrollo urbano sostenible, haciendo frente a la pobreza e identificar y afrontar desafíos nuevos y emergentes como cambio climático y el uso del agua.

El Instituto Nacional de Estadística y Censos (INEC), mediante la Dirección de Estadísticas Agropecuarias y Ambientales difunde encuestas ambientales actualizadas a nivel nacional a través del portal Vdatos, que integra la información de seis instituciones: Ministerio del Ambiente, Ministerio de Agricultura, Ganadería, Acuacultura y Pesca, Consejo Nacional de Electricidad, Instituto Nacional de Meteorología e Hidrología, Secretaría de Ambiente y Banco Central del Ecuador (INEC, 2015).

El propósito de la presente investigación es determinar el perfil de los hogares ecuatorianos respecto a los hábitos y prácticas ambientales de sus miembros, utilizando las bases de datos generadas por el INEC, mediante un análisis estadístico descriptivo y multidimensional.

El consumidor responsable es aquel que considera aspectos del entorno natural, sociales y éticos en sus decisiones de compra y consumo (Román \& Manuera, 2005). Harper \& Makatouni (2002) señalan que ser un consumidor responsable significa comprar productos que no sean dañinos para el ambiente o la sociedad. Berry \& McEachern (2005) identifican varios criterios que distinguen el consumo responsable: minimiza el consumo y evita el despilfarro, está interesado por el origen del producto y a quien se beneficia con su compra, mantiene su compromiso con el medioambiente (reduce, recicla y reusa), está motivado por la postura ética, consideran dónde y cómo se ha fabricado el producto.

En esa línea de razonamiento, Rodríguez-García (2010) destaca a la moderación como una virtud principal en el consumo, definida como el uso proporcionado de recursos que corresponde a los estándares impuestos por la práctica misma del consumo, por las preferencias personales y por influencia de la comunidad en donde interactúa. Agrega, que la moderación en el consumo es una condición adecuada entre dos extremos: el sobreconsumo y el subconsumo. El sobreconsumo incluye comportamientos nocivos como la avidez alimenticia, obsesión con los bienes materiales y el desperdicio. El subconsumo incluye conductas que limitan la plenitud humana, tales como el egoísmo, la anorexia y la avaricia.

Rodríguez-García (2010) concluye que los consumidores pueden cambiar sus decisiones de consumo y dicho cambio puede provocar, en consecuencia, que el consumidor sea más o menos responsable. Cowe \& Willams (2000) expresan, además, que el consumo responsable no se limita a la compra de unos productos 
particulares considerados éticos, o participar en causas políticas o sociales, sino que se extiende a todo tipo de prácticas, las que propician una vida integral de bienestar individual y colectivo.

\section{MATERIALES Y MÉTODOS}

En este estudio se investigó el perfil de los hogares ecuatorianos y sus prácticas ambientales, utilizando 33.033 registros de la base de datos de la encuesta nacional Prácticas Ambientales en los Hogares, realizada por el INEC el 2015.

Se efectuó el análisis estadístico descriptivo, con el fin de mostrar las frecuencias y los porcentajes de 27 variables analizadas. Se aplicó el método de análisis factorial de correspondencias múltiples, para determinar cómo los hogares son diferenciados según sus respuestas sobre prácticas ambientales (Stefos \& Koulianidi, 2016) y el método de análisis jerárquico para analizar la relación existente entre variables similares (Greenacre, 2008) permitiendo definir las clases de hogares.

El manejo de la base de datos y el análisis estadístico descriptivo se realizó utilizando el programa IBM SPSS Statistics v.20, ofrecido por la Pontificia Universidad Católica del Ecuador y el análisis estadístico multidimensional con el programa SPAD v.5.0 proporcionado por la Facultad de Humanidades de la Universidad de Egeo (Paredes, Sarmiento, \& Stefos, 2016).

\section{RESULTADOS Y DISCUSIÓN}

\section{Resultados del análisis descriptivo Tabla 1. Área de ubicación de los hogares ecuatorianos}

Tabla 1. Área de ubicación de los hogares ecuatorianos

\begin{tabular}{|l|c|c|}
\multicolumn{1}{|c|}{$\mathbf{n}$} & $\%$ \\
\hline Urbana & 18429 & $61.4 \%$ \\
\hline Rural & 11604 & $38.6 \%$ \\
\hline Total & 30033 & $100.0 \%$ \\
\hline
\end{tabular}

Del total de encuestados, el porcentaje de mayor concentración se encuentra en zonas urbanas teniendo un $61.4 \%$, a diferencia de la zona rural que presenta un $38.6 \%$.

Tabla 2. Cumplimiento de horarios en la recolección de basura y clasificación de los residuos

\begin{tabular}{|l|c|c|c|c|}
\multicolumn{2}{|c}{$\begin{array}{c}\text { Sacan la basura a } \\
\text { las horas previstas } \\
\text { de recolección }\end{array}$} & $\begin{array}{c}\text { Clasifican los } \\
\text { residuos en su hogar }\end{array}$ \\
\hline & $n$ & $\%$ & $n$ & $\%$ \\
\hline $\mathrm{Si}$ & 21699 & $72.3 \%$ & 12264 & $40.8 \%$ \\
\hline No & 2725 & $9.1 \%$ & 17769 & $59.2 \%$ \\
\hline No aplica & 5609 & $18.7 \%$ & 0 & $0 \%$ \\
\hline Total & 30033 & $100.0 \%$ & 30033 & $100.0 \%$ \\
\hline
\end{tabular}

El mayor porcentaje de las personas afirman haber sacado la basura a horas previstas de la recolección, sin embargo, el 60\% aproximadamente no clasifica los residuos. 
Tabla 3. Principal razón por la que no clasifican la basura

\begin{tabular}{|l|c|c|}
\multicolumn{2}{|c|}{ n } & $\%$ \\
\hline $\begin{array}{l}\text { No hay contenedores específicos o centros } \\
\text { de acopio para reciclables }\end{array}$ & 7047 & $23.46 \%$ \\
\hline No sabe clasificar & 4173 & $13.89 \%$ \\
\hline No conoce los beneficios & 2596 & $8.64 \%$ \\
\hline $\begin{array}{l}\text { No confía en el sistema de recolección de } \\
\text { basura }\end{array}$ & 1526 & $5.08 \%$ \\
\hline No le interesa & 2427 & $8.08 \%$ \\
\hline No aplica & 12264 & $40.84 \%$ \\
\hline Total & 30033 & $100.00 \%$ \\
\hline
\end{tabular}

El 23.5\% de hogares no clasifica la basura porque no hay contenedores específicos o centros de acopio para reciclables, un pequeño porcentaje no confía en el sistema de recolección de basura de su zona.

Tabla 4. Forma de eliminación de desechos electrónicos/eléctricos

\begin{tabular}{|l|c|c|}
\hline \multicolumn{2}{|c|}{ n } & $\%$ \\
\hline Centro de acopio o Contenedor especial & 514 & $1.7 \%$ \\
\hline Guardó, vendió, regaló & 2515 & $8.4 \%$ \\
\hline Depositó con el resto de la basura & 9948 & $33.1 \%$ \\
\hline $\begin{array}{l}\text { Quemó, enterró, botó en la quebrada, } \\
\text { desagüe }\end{array}$ & 780 & $2.6 \%$ \\
\hline No aplica & 16276 & $54.2 \%$ \\
\hline Total & 30033 & $100.0 \%$ \\
\hline
\end{tabular}

La mayoría de personas afirman eliminar los desechos electrónicos junto con la basura, solo el 1.7\% entrega en un centro de acopio o contenedor especial. 
Tabla 5. Forma de eliminación de aceite y/o grasas

\begin{tabular}{|l|c|c|}
\hline \multicolumn{2}{|c|}{$n$} & $\%$ \\
\hline Centro de acopio o Contenedor especial & 345 & $1.1 \%$ \\
\hline Guardó, vendió, regaló & 255 & $0.8 \%$ \\
\hline Quemósitó conterró, botó en la quebrada, desagüe & 6082 & $47.5 \%$ \\
\hline No generó estos desechos & 14279 & $20.3 \%$ \\
\hline $\begin{array}{l}\text { Alimento animales (solo para aceites y/o gra- } \\
\text { sas) }\end{array}$ & 7697 & $25.6 \%$ \\
\hline Total & 30033 & $100.0 \%$ \\
\hline
\end{tabular}

Aproximadamente el 50\% de personas depositan el aceite y/o grasas con el resto de la basura y desconocen métodos alternativos para formas de reciclaje. Tan solo el 1.1\% deja el aceite y/o grasas en un centro de acopio o contenedor especial.

Tabla 6. Forma de eliminación de focos ahorradores.

\begin{tabular}{|l|c|c|}
\hline \multicolumn{2}{|c|}{$n$} & $5.2 \%$ \\
\hline Centro de acopio o Contenedor especial & 1568 & $4.4 \%$ \\
\hline Guardó, vendió, regaló & 1333 & $69.6 \%$ \\
\hline Depositó con el resto de la basura & 20912 & $6.7 \%$ \\
\hline Quemó, enterró, botó en la quebrada, desagüe & 2024 & $14.0 \%$ \\
\hline No aplica & 4196 & $100.0 \%$ \\
\hline Total & 30033 & \\
\hline
\end{tabular}

La mayoría de hogares depositan los focos ahorradores con el resto de la basura, apenas el $5.2 \%$ los dejan en un centro de acopio o contenedor especial. 
Tabla 7. Reutilización del agua

\begin{tabular}{|l|c|c|}
\multicolumn{3}{c}{ n } \\
\hline Si & 11370 & $37.9 \%$ \\
\hline No & 18091 & $60.2 \%$ \\
\hline No aplica & 572 & $1.9 \%$ \\
\hline Total & 30033 & $100.0 \%$ \\
\hline
\end{tabular}

El $60.2 \%$ de los encuestados no reutilizan el agua en sus hogares, mientras que el 37.9\% afirman reusarla.

Tabla 8. Cuidado con el agua de grifo

\begin{tabular}{l|c|c|c|c|}
\multicolumn{4}{c}{$\begin{array}{c}\text { Utilizan balde en lugar de } \\
\text { manguera para ciertas actividades }\end{array}$} & $\begin{array}{c}\text { Cierran las llaves mientras } \\
\text { jabonan los platos, se bañan, o } \\
\text { se cepillan los dientes }\end{array}$ \\
\hline & $\mathbf{n}$ & $\%$ & $\mathbf{n}$ & $\%$ \\
\hline Si & 13766 & $45.8 \%$ & 22907 & $76.3 \%$ \\
\hline No & 13958 & $46.5 \%$ & 4015 & $13.4 \%$ \\
\hline No aplica & 2309 & $7.7 \%$ & 3111 & $10.4 \%$ \\
\hline Total & 30033 & $100.0 \%$ & 30033 & $100.0 \%$ \\
\hline
\end{tabular}

La proporción de personas que utilizan balde en lugar de manguera para ciertas actividades y las que no, es bastante similar. Existe un alto porcentaje de personas que se preocupan por cerrar las llaves mientras realizan algunas actividades cotidianas.

Tabla 9. Revisión regular de las tuberías y cuidado del agua

\begin{tabular}{|l|c|c|c|c|}
\hline \multicolumn{2}{|c}{$\begin{array}{c}\text { Revisan regularmente } \\
\text { sus tuberias }\end{array}$} & $\begin{array}{c}\text { Disponen de } \\
\text { economizadores de } \\
\text { chorro }\end{array}$ \\
& $\mathbf{n}$ & $\%$ & $n$ & $\%$ \\
\hline Si & 13697 & $45.6 \%$ & 2308 & $7.7 \%$ \\
\hline No & 13230 & $44.1 \%$ & 24589 & $81.9 \%$ \\
\hline No aplica & 3106 & $10.3 \%$ & 3136 & $10.4 \%$ \\
\hline Total & 30033 & $100.0 \%$ & 30033 & $100.0 \%$ \\
\hline
\end{tabular}

Los hogares que revisan regularmente las tuberías se equiparan con los que no, pero un porcentaje muy alto no disponen de economizadores de chorro. 
Tabla 10. Cuidado de agua en los inodoros.

\begin{tabular}{l|c|c|c|c|c|}
\multicolumn{4}{c}{} & $\begin{array}{c}\text { Disponen de inodoro } \\
\text { doble descarga }\end{array}$ & $\begin{array}{c}\text { Colocan una botella de agua u } \\
\text { otro objeto dentro del tanque del } \\
\text { inodoro }\end{array}$ \\
\hline Si & n & $\%$ & n & $\%$ \\
\hline No & 1198 & $4.0 \%$ & 891 & $3.0 \%$ \\
\hline No aplica & 25531 & $85.0 \%$ & 25846 & $86.1 \%$ \\
\hline Total & 3304 & $11.0 \%$ & 3296 & $11.0 \%$ \\
\hline
\end{tabular}

El 85\% de hogares no disponen de inodoro de doble descarga, tampoco colocan una botella de agua dentro del tanque para ahorrar agua.

Tabla 11. Facturación de electricidad del mes pasado

\begin{tabular}{|c|c|c|}
\hline & $n$ & $\%$ \\
\hline$[0,20]$ & 18998 & $63.3 \%$ \\
\hline$(20,40]$ & 6517 & $21.7 \%$ \\
\hline$(40,60]$ & 1090 & $3.6 \%$ \\
\hline$(60,80]$ & 239 & $0.8 \%$ \\
\hline$(80,100]$ & 102 & $0.3 \%$ \\
\hline$>100$ & 161 & $0.5 \%$ \\
\hline No aplica & 2926 & $9.7 \%$ \\
\hline Total & 30033 & $100.0 \%$ \\
\hline
\end{tabular}

El mayor porcentaje de familias encuestadas pagaron menos de veinte dólares por consumo de electricidad, durante el mes pasado.

Tabla 12. Cuidado de la energía eléctrica.

\begin{tabular}{|l|c|c|c|c|c|c|}
\hline \multicolumn{4}{|c}{$\begin{array}{c}\text { Desconectan } \\
\text { aparatos eléctricos y } \\
\text { electrodomésticos cuando } \\
\text { no los usan }\end{array}$} & $\begin{array}{c}\text { Apagan los focos al salir } \\
\text { de una habitación }\end{array}$ & $\begin{array}{c}\text { Planchan, la mayor } \\
\text { cantidad de ropa posible, } \\
\text { en una sola vez }\end{array}$ \\
& $\mathbf{n}$ & $\%$ & $\mathbf{n}$ & $\%$ & $\mathbf{n}$ & $\%$ \\
\hline Si & 20642 & $68.7 \%$ & 28263 & $94.1 \%$ & 10866 & $36.2 \%$ \\
\hline No & 8107 & $27.0 \%$ & 1239 & $4.1 \%$ & 14796 & $49.3 \%$ \\
\hline No aplica & 1284 & $4.3 \%$ & 531 & $1.8 \%$ & 4371 & $14.6 \%$ \\
\hline Total & 30033 & $100.0 \%$ & 30033 & $100.0 \%$ & 30033 & $100.0 \%$ \\
\hline
\end{tabular}

La mayoría de familias si desconectan sus equipos electrónicos para ahorrar energía. Casi la totalidad de la muestra apagan los focos con el mismo fin; sin embargo, muy pocas familias tienen la costumbre de planchar la mayor cantidad de ropa, en una sola vez. 
Tabla 13. Ahorro de energía eléctrica

\begin{tabular}{|l|c|c|c|c|} 
& \multicolumn{2}{c}{$\begin{array}{c}\text { Abren cortinas y } \\
\text { persianas para } \\
\text { aprovechar la luz de sol }\end{array}$} & \multicolumn{2}{c}{$\begin{array}{c}\text { Disponen de aparatos } \\
\text { electrodomésticos } \\
\text { ahorradores de energía }\end{array}$} \\
\hline & $\mathbf{n}$ & $\%$ & $\mathbf{n}$ & $\%$ \\
\hline $\mathrm{Si}$ & 24859 & $82.8 \%$ & 5013 & $16.7 \%$ \\
\hline No & 4339 & $14.4 \%$ & 22193 & $73.9 \%$ \\
\hline No aplica & 835 & $2.8 \%$ & 2827 & $9.4 \%$ \\
\hline Total & 30033 & $100.0 \%$ & 30033 & $100.0 \%$ \\
\hline
\end{tabular}

Un alto porcentaje de familias aprovechan la luz del sol para iluminar sus casas; aunque no disponen de electrodomésticos ahorradores de energía.

Tabla 14. Garantía ecológica, consumo y ahorro energético

\begin{tabular}{|c|c|c|c|c|}
\hline & \multicolumn{2}{|c|}{$\begin{array}{c}\text { Al momento de comprar un } \\
\text { nuevo producto, a usted le } \\
\text { importa la etiqueta de garantía } \\
\text { ecológica }\end{array}$} & \multicolumn{2}{|c|}{$\begin{array}{c}\text { Al momento de comprar un nuevo produc- } \\
\text { to, a usted le importa el consumo y ahorro } \\
\text { energético }\end{array}$} \\
\hline & $n$ & $\%$ & $n$ & $\%$ \\
\hline Nada & 7902 & $26.3 \%$ & 7546 & $25.1 \%$ \\
\hline Poco & 13958 & $46.5 \%$ & 11668 & $38.9 \%$ \\
\hline Mucho & 8173 & $27.2 \%$ & 10819 & $36.0 \%$ \\
\hline Total & 30033 & $100.0 \%$ & 30033 & $100.0 \%$ \\
\hline
\end{tabular}

Aproximadamente a la mitad de las personas encuestadas les importa "Poco" el fijarse en la etiqueta de garantía ecológica, el consumo y ahorro energético de los productos que compran.

Tabla 15. Campañas y organizaciones de protección del medio ambiente.

\begin{tabular}{|l|c|c|c|c|}
\hline \multicolumn{4}{c}{$\begin{array}{c}\text { Conoce de alguna campaña relativa } \\
\text { a la protección del medio ambiente }\end{array}$} & $\begin{array}{c}\text { Colaboran con una organización en } \\
\text { defensa del medio ambiente }\end{array}$ \\
\hline & $\mathbf{n}$ & $\%$ & $n$ & $\%$ \\
\hline $\mathrm{Si}$ & 6717 & $22.4 \%$ & 1505 & $5.0 \%$ \\
\hline No & 23316 & $77.6 \%$ & 28528 & $95.0 \%$ \\
\hline Total & 30033 & $100.0 \%$ & 30033 & $100.0 \%$ \\
\hline
\end{tabular}

La mayoría de personas encuestadas desconocen la existencia de campañas relativas a la protección del ambiente, casi la totalidad de ellos no colaboran con organizaciones en defensa del mismo. 
Tabla 16. Actitud frente a problemas ambientales

\begin{tabular}{|l|c|c|c|c|c|c|}
\hline \multicolumn{2}{|c}{$\begin{array}{c}\text { Han participado } \\
\text { en voluntariados } \\
\text { ambientales }\end{array}$} & $\begin{array}{c}\text { Se han manifestado } \\
\text { contra alguna situación } \\
\text { perjudicial }\end{array}$ & $\begin{array}{c}\text { Denuncia personalmente un } \\
\text { problema ambiental }\end{array}$ \\
\hline & $\mathbf{n}$ & $\%$ & $\mathbf{n}$ & $\%$ & $\mathbf{n}$ & $\%$ \\
\hline $\mathrm{Si}$ & 1499 & $5.0 \%$ & 1034 & $3.4 \%$ & 970 & $3.2 \%$ \\
\hline No & 28534 & $95.0 \%$ & 28999 & $96.6 \%$ & 29063 & $96.8 \%$ \\
\hline Total & 30033 & $100.0 \%$ & 30033 & $100.0 \%$ & 30033 & $100.0 \%$ \\
\hline
\end{tabular}

Prácticamente, a la totalidad de la muestra no le interesa involucrarse en temas ambientales, no pertenecen a voluntariados, no manifiestan su inconformidad con situaciones perjudiciales, ni denuncian problemas ambientales.

\section{Resultados del análisis factorial}

Los principales factores que diferencian a los hogares ecuatorianos según sus hábitos y prácticas ambientales son:

Factor uno de diferenciación (porcentaje de inercia 11,86\%): En el primer eje, por un lado, hay hogares del área urbana donde habitualmente cierran las llaves mientras jabonan los platos, se bañan o se cepillan los dientes, no disponen de inodoro doble descarga, no colocan una botella de agua u otro objeto dentro del tanque y no disponen de economizadores de chorro. Por otro lado, corresponden a hogares del área rural que principalmente eliminan los desechos eléctricos, electrónicos y focos ahorradores quemando, enterrando, botando en la quebrada o en el desagüe.

Factor dos de diferenciación (porcentaje de inercia 5,84\%): En el segundo eje, por un lado, hay hogares que habitualmente no revisan las tuberías, no disponen de economizadores de chorro, no disponen de inodoro doble descarga y ningún miembro de la familia participó en alguna organización en defensa del medio ambiente. Por otro lado, hay hogares donde a los miembros de la familia al momento de comprar un nuevo producto les importa mucho la etiqueta, la garantía ecológica, el consumo o el ahorro energético; además, durante los 12 últimos meses al menos un miembro del hogar colaboró con alguna organización en defensa del medio ambiente, participó como voluntario ambiental y se manifiestan contra alguna situación perjudicial.

Factor tres de diferenciación (porcentaje de inercia 4,64\%): En el tercer eje, por un lado, hay hogares del área urbana que durante los últimos 12 meses no clasifican los residuos, principalmente depositan con el resto de la basura los desechos electrónicos, eléctricos, aceites, grasas y focos ahorradores. Por otro lado, hay hogares del área rural que durante los últimos 12 meses, clasificaron los residuos, utilizaron los desechos de aceites y grasas como alimento para animales.

\section{Resultados del análisis jerárquico}

El análisis jerárquico permitió definir los siguientes grupos de hogares según sus hábitos y prácticas ambientales (Figura 1):

Primer grupo (11092 hogares, 36,93\% de la muestra): Los hogares de este grupo son del área urbana, no clasifican los residuos porque no hay contenedores específicos o centros de acopio para reciclables. Principalmente depositan con el resto de basura los desechos de cocina (aceites y grasas) y focos ahorradores. 
Segundo grupo (5656 hogares, 18,83\% de la muestra): Los hogares de este grupo son del área rural, en el momento de comprar un nuevo producto nada les importa la etiqueta, la garantía ecológica, el consumo o el ahorro energético. No revisan regularmente las tuberías ni disponen de economizadores de chorro.

Figura 1. Análisis jerárquico

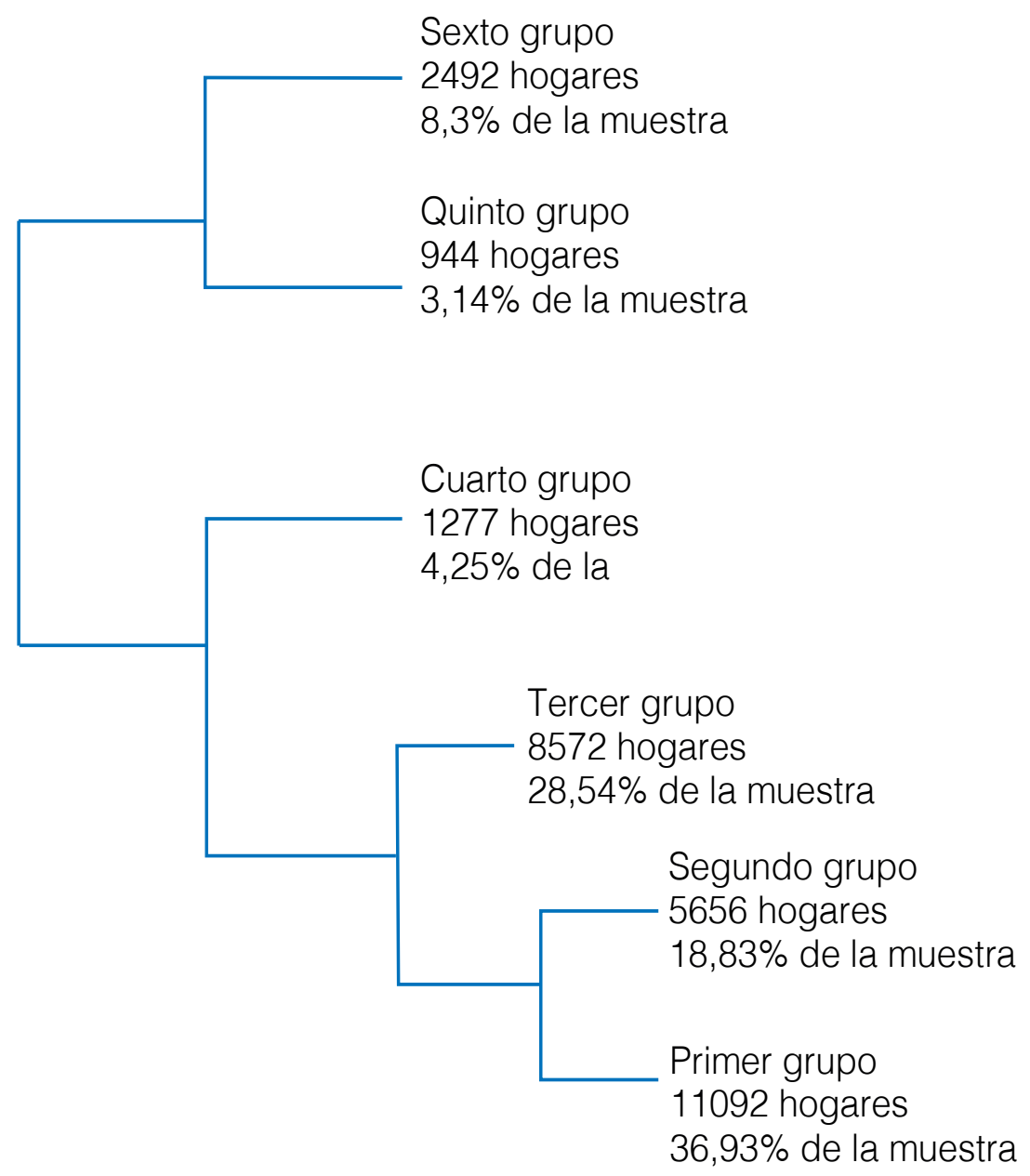

Tercer grupo (8572 hogares, $\mathbf{2 8 , 5 4 \%}$ de la muestra): Los hogares de este grupo son del área urbana, si clasifican los residuos, habitualmente cierran las llaves mientras jabonan los platos, se bañan o se cepillan los dientes, sacan la basura a las horas previstas de recolección, eliminan los focos ahorradores entregando a un centro de acopio o depositando en un contenedor especial.

Cuarto grupo (1277 hogares, 4,25\% de la muestra): Los miembros de estos hogares, colaboran con organizaciones en defensa del ambiente, participan como voluntarios ambientales, se manifiestan contra cualquier situación perjudicial y denuncian personalmente algún problema ambiental. Manifiestan tener conocimiento de alguna campaña relativa a la protección del ambiente en los últimos 12 meses.

Quinto grupo (944 hogares, 3,14\% de la muestra): Los hogares de este grupo son del área rural, al momento de comprar un nuevo producto nada les importa la etiqueta, la garantía ecológica, el consumo o el ahorro energético. Manifiestan que no generan desechos de cocina (aceites y grasas) y no saben clasificar los desechos. 
Sexto grupo (2492 hogares, $\mathbf{8 , 3 \%}$ de la muestra): Los hogares de este grupo son del área rural, que principalmente eliminan los desechos eléctricos, electrónicos y focos ahorradores quemando, enterrando, botando en la quebrada o en el desagüe. Manifiestan que no generan desechos de cocina (aceites y grasas) y no saben clasificar los desechos. En la Figura 2 se presentan los centroides de los seis grupos antes descritos según los ejes factoriales 1 y 2.

Figura 2. Análisis de correspondencias

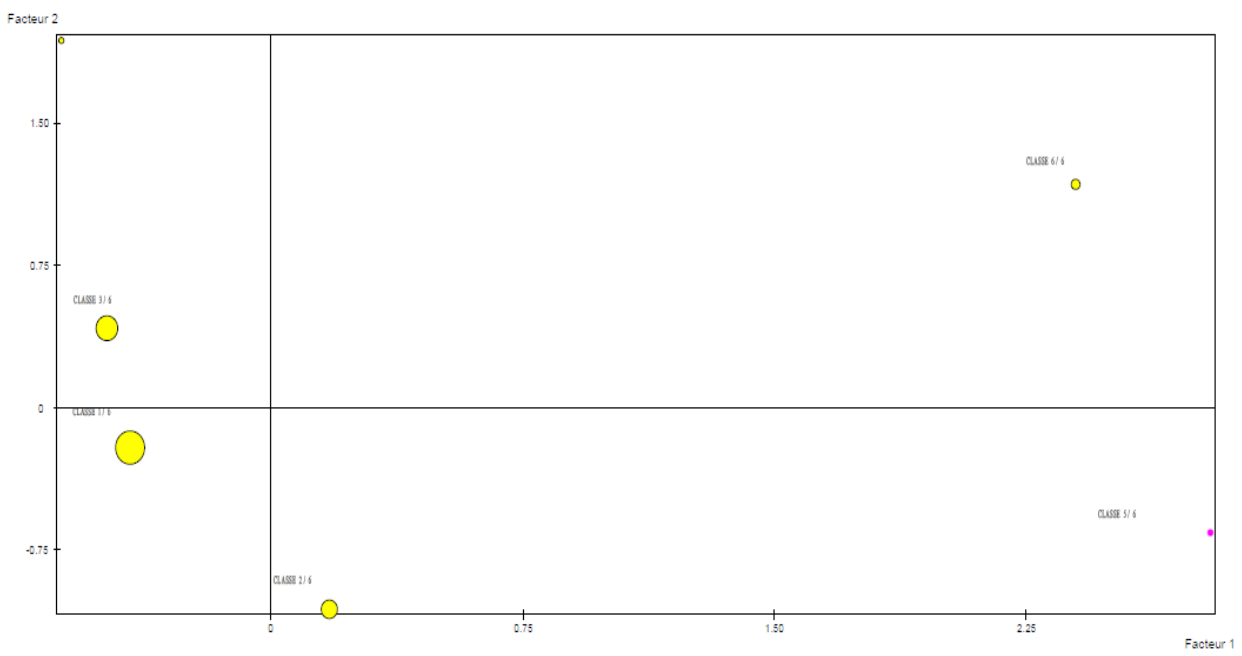

\section{CONCLUSIONES}

Los resultados que sobresalen en el estudio descriptivo y son confirmados por el análisis factorial hacen referencia a las siguientes variables: área de ubicación, clasificación de los residuos, forma de eliminar los residuos, hábitos e instalaciones para ahorro del agua.

El porcentaje de hogares del área urbana y rural es $61.4 \%$ y $38.6 \%$ respectivamente. El $59.2 \%$ de hogares no clasifican los residuos, porque no hay contenedores específicos o centros de acopio para reciclables (23.5\%) o no sabe clasificar (13.9\%). El 33.1\% de hogares depositaron los desechos electrónicos/eléctricos con el resto de basura, el $47.5 \%$ eliminó el aceite y/o grasas de cocina con el resto de basura y el $69.6 \%$ eliminó los focos ahorradores con el resto de basura. El 76.3\% de hogares cierran las llaves mientras jabonan los platos, se bañan y se cepillan los dientes. El 7.7\% de hogares disponen de economizadores de chorro, el $4 \%$ disponen de inodoro doble descarga y el $3 \%$ colocan una botella de agua u otro objeto dentro del tanque.

El primer grupo jerárquico es el más representativo con el 36.93\% de hogares, cuyo perfil es: hogares del área urbana, no clasifican los residuos porque no hay contenedores específicos o centros de acopio para reciclables y depositan con el resto de basura los desechos de cocina (aceites y grasas) y focos ahorradores. El segundo grupo corresponde al 18,8\% de hogares, cuyo perfil es: hogares del área rural, al momento de comprar un nuevo producto nada les importa la etiqueta, la garantía ecológica, el consumo o el ahorro energético; y no revisan regularmente las tuberías ni disponen de economizadores de chorro.

El tercer grupo con el 28,5\% de hogares, corresponden a: hogares del área urbana, clasifican los residuos, habitualmente cierran las llaves mientras jabonan los platos, se bañan o se cepillan los dientes, sacan la basura a las horas previstas de recolección, eliminan los focos ahorradores entregando a un centro de acopio o depositando en un contenedor especial. Los otros grupos tienen bajos porcentajes de representación. 
Siguiendo el planteamiento de Rodríguez-García (2010) de que los consumidores pueden cambiar sus hábitos, como resultado de sus decisiones de consumo, y dicho cambio puede resultar en que el consumidor sea más o menos responsables, se puede concluir que los hogares ecuatorianos no tienen patrones de prácticas ambientales responsables. Por lo cual, es imperativo que los estamentos gubernamentales y seccionales, las instituciones educativas y la sociedad civil organizada, emprendan campañas dirigidas a la concientización de las buenas prácticas de consumo responsable, de esta forma se tendría una sociedad ecuatoriana caracterizada por su conciencia ambiental.

\section{REFERENCIAS BIBLIOGRÁFICAS}

Berry, H., \& McEachern, M. (2005). Informing ethical consumers. The Ethical Consumer. London. https://doi. org/9781412903530

CEPAL. (2015). El desafío de la sostenibilidad ambiental en América Latina y el Caribe. (Naciones Unidas, Ed.). Santiago de Chile.

Cowe, R., \& Willams, S. (2000). Who are the ethical consumers? Ethical Consumerism Report.

Greenacre, M. (2008). La práctica del análisis de correspondencias. (R. Editorial, Ed.), Statewide Agricultural Land Use Baseline 2015 (1). Bilbao: Valant 2003.

Harper, G., \& Makatouni, A. (2002). Consumer perception of organic food production and farm animal welfare. British Food Journal, (104)(3/4/5 287-299).

INEC. (2015). Prácticas Ambientales en los Hogares - 2015.

ONU. (2013). Desarrollo sostenible en América Latina y el Caribe: Seguimiento de la agenda de las Naciones Unidas para el desarrollo post-2015 y Río+20.

Paredes, A., Sarmiento, M., \& Stefos, E. (2016). Perfil de los desempleados ecuatorianos. Un análisis multidimensional. Revista PUCE, (102), 325-367.

PNUMA. (2011). Eficiencia en el uso de los recursos en América Latina: Perspectivas e implicancias económicas. (R. M. PNUMA, Ed.). Panamá.

Rodríguez-García, D. (2010). Beyond assimilation and multiculturalism: A critical review of the debate on managing diversity. Journal of International Migration and Integration, (11)(3), 251-271. https://doi.org/ DOI 10.1007/s12134-010-0140-x

Román, S., \& Manuera, J. (2005). Determinants and consequences of ethical behaviour: an empirical study of salespeople. European Journal of Marketing, 39(5/6), 473-495. https://doi. org/10.1108/030905660510590674

Stefos, E., \& Koulianidi, G. (2016). 2016_STEFOS_Uso de software R.pdf (pp. 57-65). Universidad de los Andes, Consejo de Publicaciones PUCE-SI sede IBARRA. 